\title{
Low-Frequency Dielectric Properties of Liquid Boric Oxide
}

\author{
Kurt H. Stern
}

(February 19, 1965)

\begin{abstract}
The dielectric constant and loss of glassy $\mathrm{B}_{2} \mathrm{O}_{3}$ have been measured from 500 to $800{ }^{\circ} \mathrm{C}$ over the frequency range 1 to $50 \mathrm{kHz}$. The material behaves essentially like a nonpolar polymer; no relaxation phenomena could be identified. Over the entire frequency and temperature range $\epsilon^{\prime}$ lies between 3.1 and 3.2. Losses increase with increasing temperature and decreasing frequency and appear to be due to ionic impurities. The activation energy for conductance is $77.8 \mathrm{~kJ}$ (18.6 kcal), independent of frequency.
\end{abstract}

\section{Introduction}

Boric oxide $\left(\mathrm{B}_{2} \mathrm{O}_{3}\right)$ is one of the two most extensively studied inorganic glass-forming oxides, the other being $\mathrm{SiO}_{2}$. Many of its physical properties have been measured as a function of temperature $[1]^{1}$ but the determination of accurate values has been hampered by the great difficulty of preparing the pure, especially water-free, compound.

One aspect of these studies is the elucidation of the vitreous structure, which has even been described in terms of $\mathrm{B}_{4} \mathrm{O}_{6}$ molecules [2], but now is generally considered to be a disordered, three-dimensional network held together by $\mathrm{B}-\mathrm{O}$ bonds which rupture increasingly as the temperature is raised. Consequently the viscosity decreases, and even at relatively moderate temperatures $\left(800-1000^{\circ} \mathrm{C}\right)$ the material flows like a viscous liquid.

Another interesting property of $\mathrm{B}_{2} \mathrm{O}_{3}$ is its solvent power for a large number of inorganic oxides [3] and salts [4]. It is this latter aspect, together with the low viscosity, which makes it possible-at least in principle-to study dilute electrolyte solutions at high temperatures. Up to now the only molecular solvent used in this temperature range has been high-pressure, supercritical water [5].

In order to interpret conductance data by the classical Debye-Hückel-Onsager methods several solvent properties must be known, principally viscosity and dielectric constant. The former has been measured over a wide temperature range [6], but low-frequency dielectric data are largely lacking. The dielectric constant $\epsilon^{\prime}$ of a sample of unspecified purity was reported to be [7] 3.2, independent of temperature from 20 to $400^{\circ} \mathrm{C}$; preliminary data by Kruh and Stern [4] between 25 and $570^{\circ} \mathrm{C}$ on a not-entirely-waterfree sample showed a marked temperature dependence

${ }^{1}$ Figures in brackets indicate the literature references at the end of this paper. which is, however, due to an error in calculation. It thus seemed of interest to determine the dielectric constant from a temperature at which liquid flow begins to as high a temperature as our apparatus would permit. This upper cutoff point is largely dependent on the losses which increase markedly with increasing temperature and decreasing frequency. In the present work we report data from 500 to $800{ }^{\circ} \mathrm{C}$.

\section{Experimental Part}

\subsection{Preparation of Anhydrous $\mathrm{B}_{2} \mathrm{O}_{3}$}

Baker Analyzed Reagent $\mathrm{H}_{3} \mathrm{BO}_{3}$ was dehydrated in a cylindrical platinum crucible of $170 \mathrm{ml}$ capacity by increasing the temperature to $800{ }^{\circ} \mathrm{C}$ over several weeks. After this preliminary dehydration the crucible was cooled and transferred to a long mullite tube. The tube was positioned upright in the furnace with the crucible at its bottom. A rubber stopper with a long quartz tube through it was placed in the tube. When the crucible had reached $1200{ }^{\circ} \mathrm{C}$ dry $\mathrm{BCl}_{3}$ gas was passed through the quartz tube and a platinum tube fastened at its lower end which projected into the melt. $\mathrm{BCl}_{3}$ was bubbled through the $\mathrm{B}_{2} \mathrm{O}_{3}$ until $\mathrm{HCl}$ evolution had ceased. It was then replaced by dry helium. The melt was finally cooled to room temperature and stored in a desiccator. This procedure removes water completely [8]. A semiquantitative spectro-chemical analysis of the $\mathrm{B}_{2} \mathrm{O}_{3}$ showed that, except for sodium, ionic impurities constituted approximately 0.01 percent.

\subsection{Cell Design and Construction}

In order to eliminate difficulties associated with lead capacitance a three-terminal method was used. The cell was of the immersion type, the entire cell assembly fitting onto the crucible containing the melt (fig. 1). 


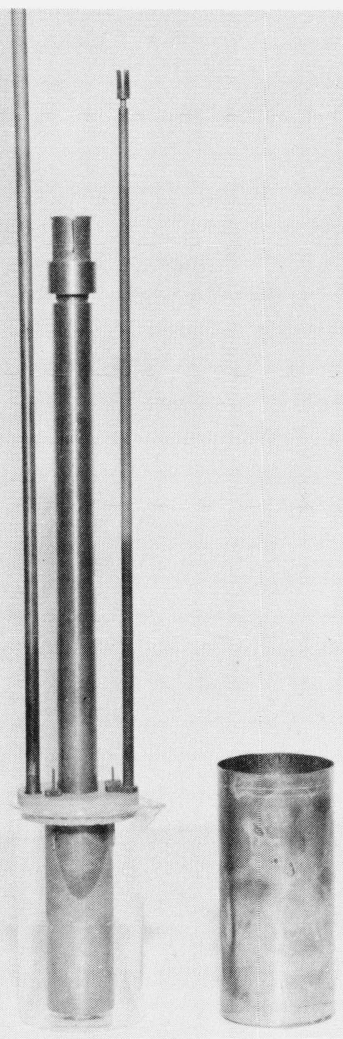

(a)

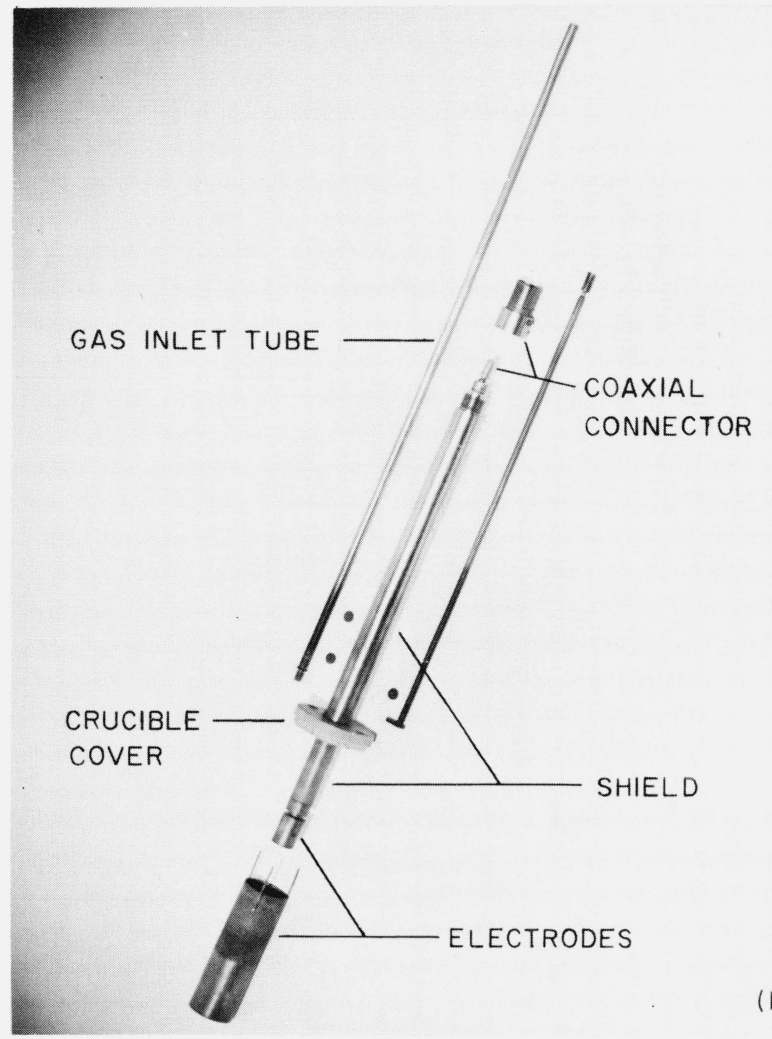

(b)

Figure 1. Dielectric Cell.

(a) Assembled Cell. The glass beaker shows the cell in upright position. Actual $\mathrm{Pt}$ beaker used on right. (b) Exploded view.

All metallic parts were made of heavy-walled nickel tubes and rods; all insulating washers, spacers, etc., were machined from lava stone and fired at a high temperature to remove volatile impurities and to fix final dimensions. This cell is easily assembled and taken apart, its capacitance remaining fixed to within $\pm 0.005 \mathrm{pF}$. A photograph of the assembled cell is shown in figure la and a partly exploded view in figure lb. Its capacitance was approximately $2.5 \mathrm{pF}$.

\subsection{Dielectric Measurements}

The platinum crucible containing the dehydrated $\mathrm{B}_{2} \mathrm{O}_{3}$ was transferred to a well-regulated furnace and heated under dry helium until the glass became sufficiently soft to permit immersion of the cell assembly described previously. A slow flow of dry helium was maintained over the melt surface. The temperature was measured with a calibrated platinum-platinum/ rhodium thermocouple whose hot junction was immersed directly in the glass. Dielectric constant and loss were measured with a General Radio Type 1615-A capacitance bridge at each of several constant temperatures between 500 and $900{ }^{\circ} \mathrm{C}$. In each measurement the frequency was varied from as low as the bridge could be balanced to a maximum of $50 \mathrm{kHz}$. With increasing temperature low-frequency dielectric losses increased so that at the higher temperatures only measurements at the higher frequencies were possible.

The cell was calibrated empty over the same temperature and frequency range as used in the measurements. Above $2 \mathrm{kHz}$ the capacitance was virtually independent of frequency, but it increased noticeably with temperature; e.g., from $2.533 \mathrm{pF}$ at $511{ }^{\circ} \mathrm{C}$ to $2.552 \mathrm{pF}$ at $785^{\circ} \mathrm{C}$.

\section{Results}

\subsection{Treatment of Experimental Data}

The quantities measured experimentally are $C_{0}$ = capacitance of the empty cell, $C_{s}=$ series capacitance of the filled cell, $D=$ dissipation factor, and frequency $f$. From these, the real and imaginary parts of the complex dielectric constant

$$
\epsilon^{*}=\epsilon^{\prime}-i \epsilon^{\prime \prime}
$$

are calculated from the well-known relations

$$
C_{p}=\frac{C_{\mathrm{s}}}{1+D^{2}}
$$




$$
\epsilon^{\prime}=C_{p} / C_{0}
$$

and the conductance

$$
G=D \omega C_{p}
$$

where

$$
\omega=2 \pi f
$$

$C_{s}$ could usually be determined to six significant figures and $D$ to four, but at high $D$ values the sensitivity of the bridge decreased. Some scatter of unknown origin reduced the precision of $\epsilon$ considerably and therefore dielectric constants are only reported graphically (fig. 3).

Although the cell cannot be calibrated as a conductance cell (the resistance varies with the depth of immersion of the electrodes in the calibrating solution) the specific conductance $\sigma$ can be calculated from the dielectric measurements as follows:

By definition

$$
G=\sigma(A / 1)
$$

where $A$ and 1 represent the area and distance of separation of the electrodes. From eq (3)

$$
C_{p}=\epsilon^{\prime} C_{0}=\epsilon^{\prime} \epsilon_{0}(A / 1)
$$

where $\epsilon_{0}$, the permittivity of free space, is $8.85 \times 10^{-14}$ $\mathrm{F} \mathrm{cm}^{-1}$. From (4), (6) and (7)

$$
\sigma=D \omega \epsilon^{\prime} \epsilon_{0}
$$

so that $(A / 1)$ does not have to be known explicitly.

\subsection{Dielectric and Conductance Data}

A plot of $\epsilon^{\prime}$ as a function of temperature at various

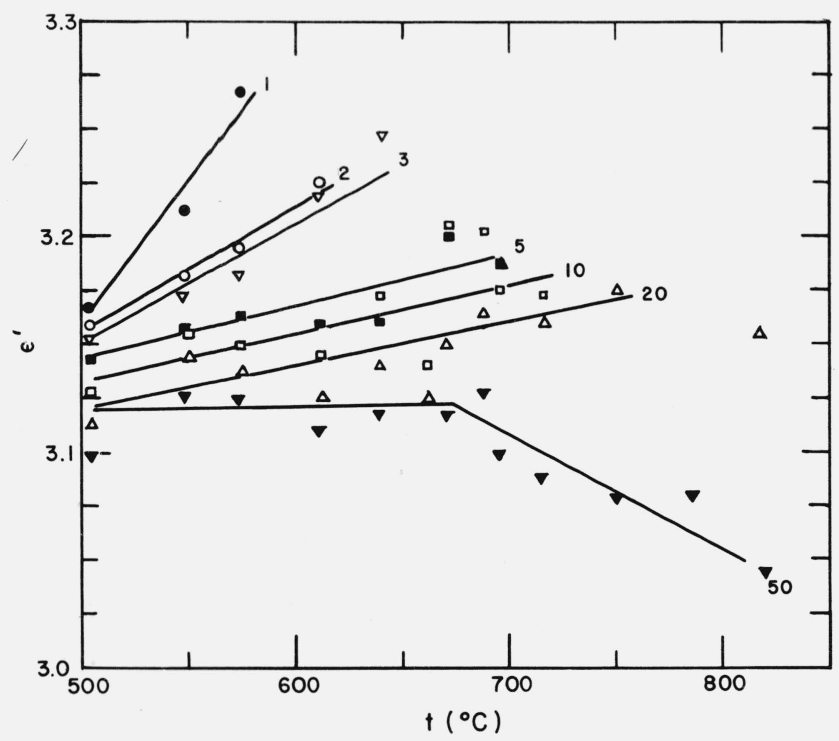

FIGURE 2. $\epsilon^{\prime}$ as a function of temperature.

Frequencies $(\mathrm{kHz})$ to the right of respective curves fixed frequencies is shown in figure 2. The large increase of $\epsilon^{\prime}$ with temperature at 1 and $2 \mathrm{kHz}$ is most likely caused by polarization effects at the electrodes due to ionic impurities in the glass. The decrease of $\epsilon^{\prime}$ at $50 \mathrm{kHz}$ above $700{ }^{\circ} \mathrm{C}$ probably reflects failure of the bridge which becomes increasingly inaccurate above $20 \mathrm{kHz}$. Although the data scatter considerably, all values of $\epsilon^{\prime}$ from 5 to $20 \mathrm{kHz}$ lie between 3.12 and 3.20 and exhibit a slight positive temperature coefficient. In figure 3 plots of $\epsilon^{\prime}$ versus $\log f$ at several temperatures are shown.

The temperature dependence of $D$ is shown explicitly for several different frequencies in figure 4. In every case $\log D$ is a linear function of temperature, the temperature coefficient $d \log D / d T$ increasing very slightly with decreasing frequency. This behavior was also observed by Strutt [9] for a number of $\mathrm{SiO}_{2}-$ $\mathrm{Na}_{2} \mathrm{O}$ glasses in which the losses are primarily conductive. If ionic conduction is the primary source of loss in $\mathrm{B}_{2} \mathrm{O}_{3}$, ln $\sigma$ would be expected to be a linear function of $1 / T$, independent of frequency. That this is indeed the case is shown in figure 5 . From the slope and the relation

$$
\sigma=A e^{-E^{\neq} / R T}
$$

the activation energy $E^{\neq}$was found to be $77.8 \mathrm{~kJ}$ $(18.6 \mathrm{kcal})$ independent of frequency, which is considerbly lower than the $26 \mathrm{kcal} /$ mole found by Mackenzie [6] for the temperature range 850 to $1350{ }^{\circ} \mathrm{C}$. An increase in $E^{\neq}$with temperature is not reasonable. It is possible, however, that the principal impurities giving rise to the conductance are different in the two cases. Ionization of $\mathrm{B}_{2} \mathrm{O}_{3}$ at this low temperature can be ruled out [6].

If the higher activation energy is associated with ionized water transport some special mechanism would have to be postulated which hinders these small ions compared to, for example, sodium. In figure 6 the frequency dependence of $\omega D \epsilon^{\prime}=\sigma / \epsilon_{o}$ is shown for the lowest three temperatures. The slight increase with increasing frequency suggests an increased freedom of movement of the ions about their equilibrium positions (short term transient response).

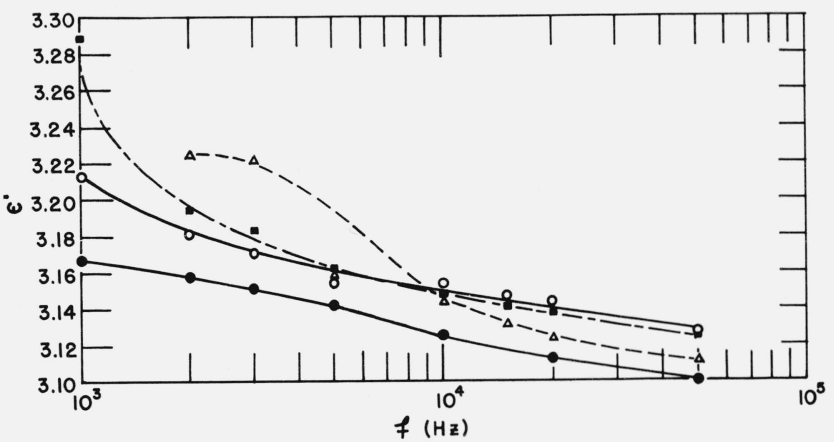

Figure 3. $\epsilon^{\prime}$ as a function of frequency.

$-0502.8^{\circ},-0-546.5^{\circ},---\square--573.8^{\circ},--\Delta-611.2^{\circ}$. 


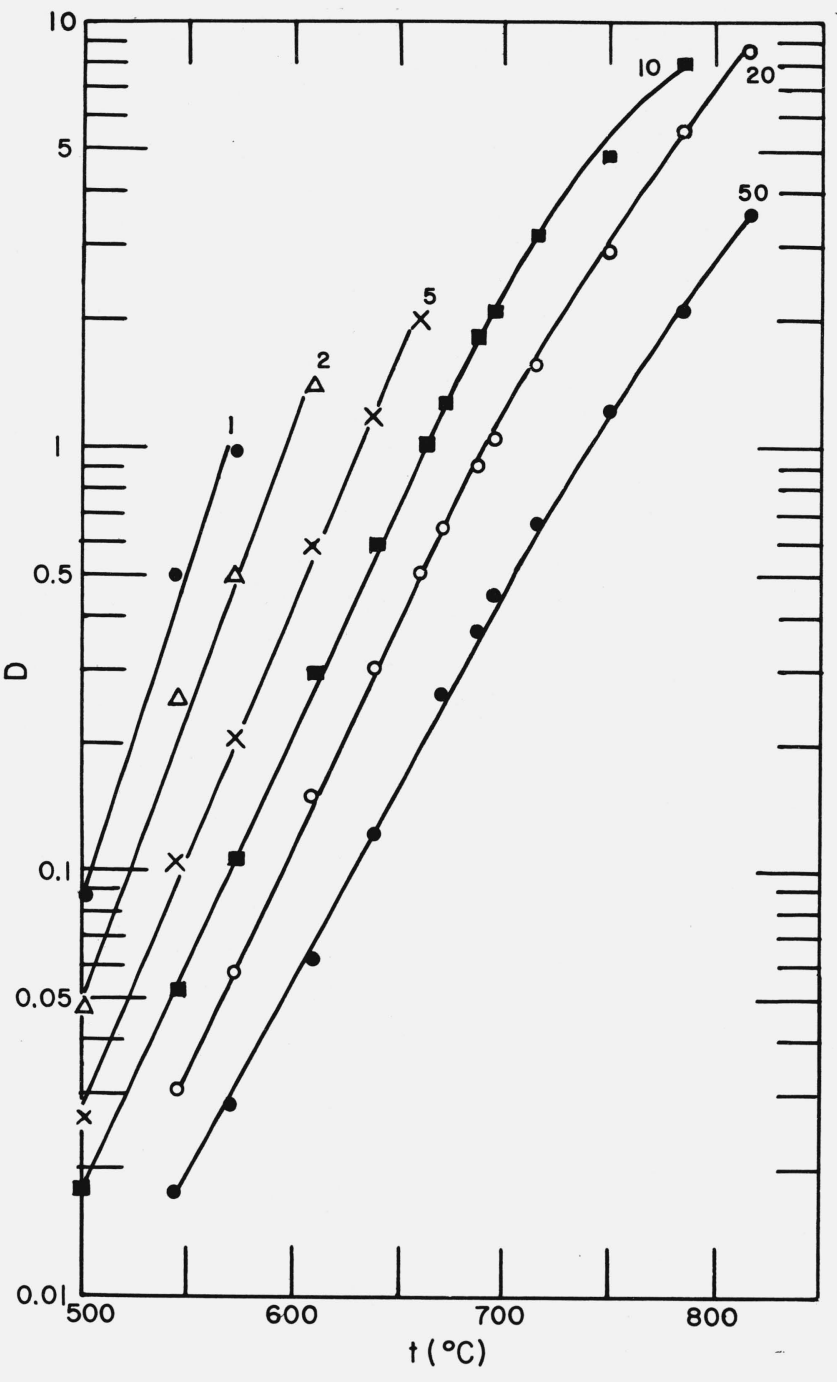

Figure 4. D as a function of temperature.

Frequencies $(\mathrm{kHz})$ shown at top of respective curves.

\section{Discussion}

The dielectric behavior of $\mathrm{B}_{2} \mathrm{O}_{3}$ found in this study is consistent with previous work on this material. The three-dimensional random network behaves essentially like a nonpolar liquid. There may be a very small dipole relaxation which could arise from a nonstoichiometric distribution of boron and oxygen atoms in some of the clusters which are free to move at elevated temperatures.

The loss appears to be entirely conductive and due to impurities, such as sodium ions, in the melt. We have, however, no good explanation for the difference of the activation energy of conductance between this work and Mackenzie's.

If the behavior of salts dissolved in $\mathrm{B}_{2} \mathrm{O}_{3}$ depends primarily on the dielectric constant of the medium, as is the case with ordinary molecular solvents, extensive ionic association into ion pairs and higher

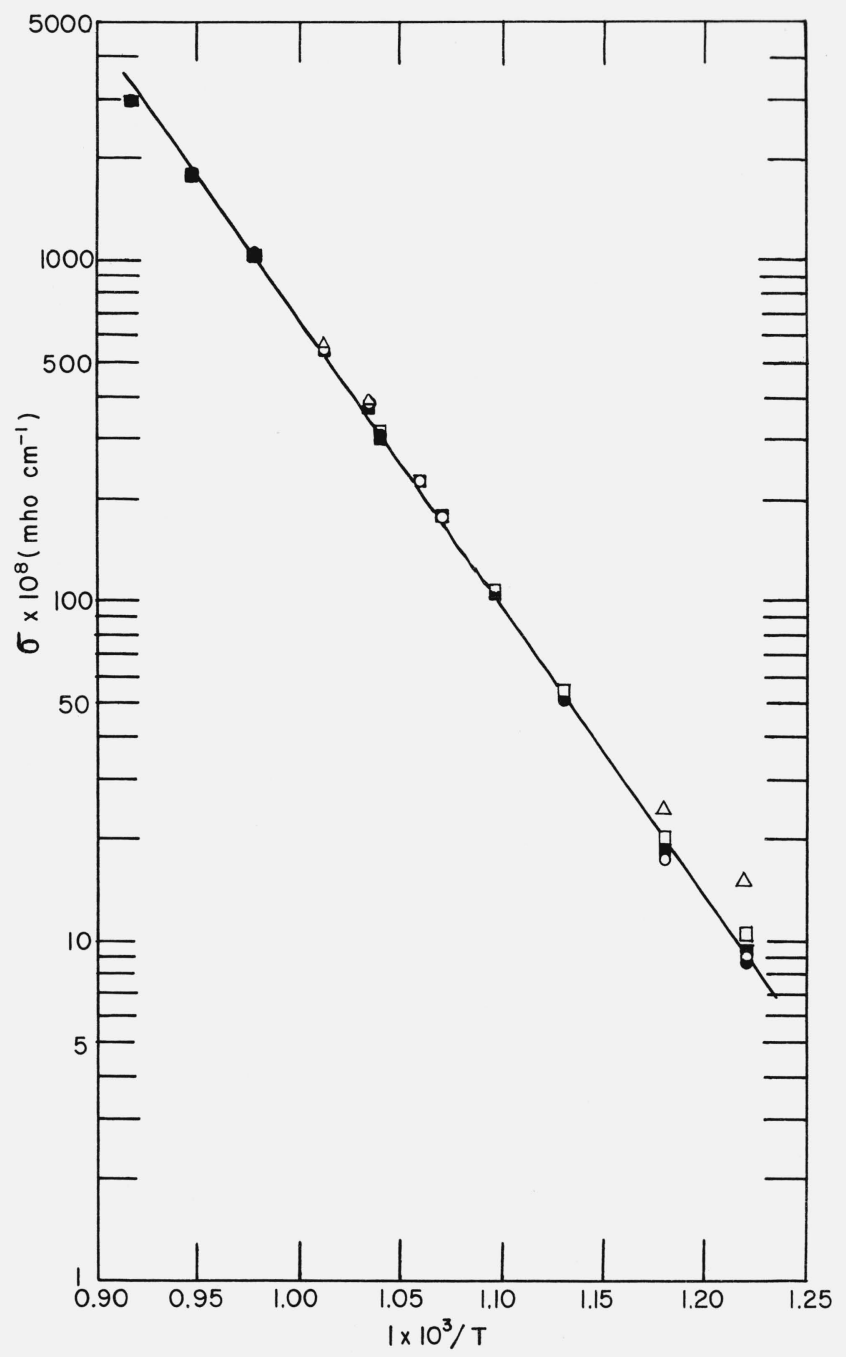

FIGURE 5. Specific conductivity $\sigma$ as a function of $1 / \mathrm{T}$.

Frequencies in kHz: $1, \bigcirc 5, \square 10, \square 20, \triangle 50$.

aggregates at even quite low concentrations would be expected. In addition $\Lambda_{0}$, the equivalent conductance at infinite dilution, should be low because of the high solvent viscosity and can be estimated from the Walden product $\Lambda_{0} \eta$ obtained for a particular electrolyte in other solvents.

For electrolytes with high-charge density anions which compete with oxygen for boron bonds [4] it is quite possible that only cations would conduct charge. It should be possible to distinguish this possibility by determining $\Lambda_{0}$ for a number of salts having the same cation. By the same method, the question of oxide ion mobility in the case of dissolved oxides could also be answered.

In any case, it is clear that for such studies the solvent must be dry since at elevated temperatures small amounts of salt can easily hydrolyze in residual water, e.g., $2 \mathrm{NaCl}+\mathrm{H}_{2} \mathrm{O}=2 \mathrm{HCl}+\mathrm{Na}_{2} \mathrm{O}$. 


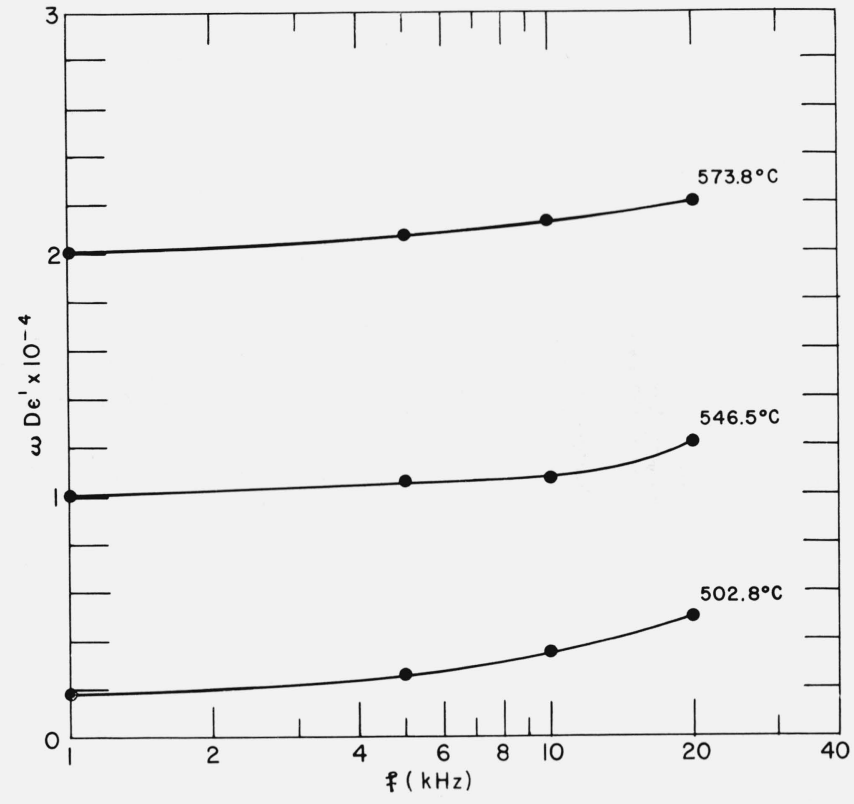

FIGURE 6. $\omega \mathrm{D} \epsilon^{\prime}=\sigma / \epsilon_{o}$ as a function of frequency.

Temperature to the right of respective curves.

A number of authors have presented conflicting evidence as to whether alkali halides react with $\mathrm{B}_{2} \mathrm{O}_{3}$ directly to produce free $\mathrm{Cl}_{2}$ and sodium borate [1]. It is conceivable that these early workers were actually noting the hydrolysis in insufficiently dried $\mathrm{B}_{2} \mathrm{O}_{3}$, but this possibility needs further investigation.
I am greatly indebted for much helpful advise concerning dielectric measurement and cell design to N. L. Brown, A. H. Scott, and W. P. Harris; and to M. Broadhurst for enlightening discussions on dielectric theory.

\section{References}

[1] $c f$. Gmelin, Handbook of Inorganic Chemistry, 8th ed., 13 supplement, pp. 125-136, Weinheim, 1954.

[2] K. Fajans and S. W. Barber, J. Am. Chem. Soc. 74, 2761(1952).

[3] M. Foex, Ann. Chim. [11], 359(1939).

[4] R. F. Kruh and K. H. Stern, J. Am. Chem. Soc. 78, 278(1956).

[5] e.g., A. S. Quist, E. U. Franck, H. R. Jolley, W. L. Marshall, J. Phys. Chem. 67, 2453(1963).

[6] J. D. Mackenzie, J. Phys. Chem. 63, 1875(1959).

[7] N. P. Begorodicki and V. N. Malysev, Zhurn. techn. Fiz. (USSR) 5, 612(1939).

[8] A. J. Harrison, J. Am. Ceram. Soc. 30, 362(1947).

[9] M. J. O. Strutt, Arch. f. Elektrotechnik 25, 715(1931).

(Paper 69A3-347) 